\title{
Fatores associados à mudança percebida pelos usuários em Centros de Atenção Psicossocial em Álcool e Outras Drogas
}

Factors associated with the user's perceived change in a Psychosocial Care Centers for Alcohol and Other Drugs

Factores asociados al cambio percibido por los usuarios en un Centro de Atención Psicosocial por Alcohol y Otras Drogas

Rejane Maria Dias de Abreu ORCID: https://orcid.org/0000-0003-1237-9946 Universidade Federal do Triângulo Mineiro, Brasil

E-mail: rejane.abreu12@gmail.com

Guilherme Correa Barbosa

ORCID: https://orcid.org/0000-0002-7433-8237 Universidade Estadual Paulista "Júlio de Mesquita Filho", Brasil E-mail: g.barbosa@unesp.br

Paula Hayasi Pinho

ORCID: https://orcid.org/0000-0001-8922-0699 Universidade Federal do Recôncavo da Bahia, Brasil e-mail: paulahpinho@gmail.com

Heloísa Garcia Claro

ORCID: https://orcid.org/0000-0003-1504-7074 Universidade Estadual de Campinas, Brasil E-mail: clarohg@unicamp.br

Maria Odete Pereira

ORCID: https://orcid.org/0000-0002-9418-2524 Universidade Federal de Minas Gerais, Brasil e-mail: m.odetepereira@gmail.com

Márcia Aparecida Ferreira de Oliveira

ORCID: https://orcid.org/0000-0002-1069-8700 Universidade de São Paulo, Brasil E-mail: marciaap@usp.br

\section{Resumo}

Objetivo: avaliar a mudança percebida e identificar os fatores a ela associados em usuários dos Centros de Atenção Psicossocial em Álcool e Outras Drogas. Método: estudo transversal com 330 usuários de 13 Centros de Atenção Psicossocial em Álcool e Outras Drogas do Estado de Minas Gerais. A percepção de mudança em função do tratamento foi identificada a partir da Escala de Mudança Percebida e a análise ajustada dos dados, realizada por modelo de regressão linear de mínimos quadrados. Resultados: a prevalência de mudança percebida foi de $86,1 \%$, com escore médio de 2,62. Fatores diretamente associados ao grau de percepção de melhora global na análise múltipla: maior escolaridade (Ensino Médio ou curso técnico completo), frequência no serviço, participação em atividades em grupo e satisfação com o serviço. As variáveis sexo feminino, consumo de bebida alcóolica, histórico de internação e intercorrência durante o tratamento demonstraram menor percepção de melhora da assistência. Conclusões: a análise dos fatores associados aos construtos avaliados indicou forte associação de variáveis socioeconômicas, clínicas, do processo e de satisfação global na determinação da mudança percebida pelos usuários, visando a modificações no cuidado que podem influenciar no envolvimento do tratamento aos usuários de substâncias psicoativas.

Palavras-chave: Avaliação em saúde; Avaliação de resultados (cuidados de saúde); Transtornos relacionados ao uso de substâncias; Serviços comunitários de saúde mental; Satisfação do paciente; Enfermagem psiquiátrica.

\begin{abstract}
Objective: Evaluate the perceived change and identify the factors associated with it in users of the Psychosocial Care Centers for Alcohol and Other Drugs. Method: a cross-sectional study with 330 users of 13 Psychosocial Care Centers for Alcohol and Other Drugs in the State of Minas Gerais. The perception of change resulting from the treatment was identified using the Perceived Change Scale and the adjusted analysis of the data carried out using the least-squares linear regression model. Results: the prevalence of perceived change was $86.1 \%$, with an average score of 2.62. Factors directly associated with the degree of perception of global improvement in multiple analysis: higher schooling (high school or complete technical course), attendance at the service, participation in group activities, and satisfaction with
\end{abstract}


the service. The variables female gender, alcohol consumption, history of hospitalization, and complications during treatment showed less perception of improvement in care. Conclusions: the analysis of the factors associated with the evaluated constructs indicated a strong association of socioeconomic, clinical, process, and global satisfaction variables in determining the change perceived by users, aiming at changes in care that can influence the treatment involvement of users of psychoactive substances.

Keywords: Health evaluation; Outcome assessment, Health care; Substance-related disorders; Community mental health services; Patient satisfaction; Psychiatric nursing.

\section{Resumen}

Objetivo: Evaluar el cambio percibido e identificar los factores asociados al mismo en usuarios de los Centros de Atención Psicosocial por Alcohol y Otras Drogas. Método: estudio transversal con 330 usuarios de 13 Centros de Atención Psicosocial por Alcohol y Otras Drogas del Estado de Minas Gerais. La percepción de cambio resultante del tratamiento se identificó mediante la Escala de Cambio Percibido y el análisis ajustado de los datos realizado mediante el modelo de regresión lineal de mínimos cuadrados. Resultados: la prevalencia de cambio percibido fue del 86,1\%, con una puntuación media de 2,62. Factores directamente asociados al grado de percepción de mejora global en el análisis múltiple: escolaridad superior (bachillerato o curso técnico completo), asistencia al servicio, participación en actividades grupales y satisfacción con el servicio. Las variables sexo femenino, consumo de alcohol, antecedentes de hospitalización y complicaciones durante el tratamiento mostraron menor percepción de mejoría en la atención. Conclusiones: el análisis de los factores asociados a los constructos evaluados indicó una fuerte asociación de variables socioeconómicas, clínicas, de proceso y de satisfacción global en la determinación del cambio percibido por los usuarios, apuntando a cambios en la atención que pueden influir en la implicación terapéutica de los usuarios de psicoactivos. sustancias.

Palabras clave: Evaluación en salud; Evaluación de resultado en la atención de salud; Trastornos relacionados con sustancias; Servicios comunitarios de salud mental; Satisfacción del paciente; Enfermería psiquiátrica.

\section{Introdução}

As conquistas advindas da Reforma Psiquiátrica implicaram diferentes ações necessárias para consolidar modelos de atenção que resgatassem a cidadania das pessoas com problemas em saúde mental, tanto na construção como na implantação de serviços substitutivos em oposição ao modelo manicomial (Miranda, Souza, Ferreira, 2014; Lapischies, Jardim, Kantorski, 2014).

$\mathrm{Na}$ área de álcool e outras drogas, a literatura evidencia que o consumo abusivo e a dependência de drogas lícitas e ilícitas constituem dois dos maiores desafios contemporâneos no que diz respeito à demanda de saúde pública mundial. Cabe destacar que não se trata somente de um problema da atenção à saúde, uma vez que dados resultantes de estudos epidemiológicos demonstram que tal desafio possui caráter não apenas nacional, mas global (WHO, 2019).

O governo brasileiro, vinha a adotar estratégias que visavam a promoção e a proteção à saúde dos consumidores de substâncias psicoativas (SPA) por meio da política do Ministério da Saúde para a atenção integral aos usuários de álcool e outras drogas (Brasil, 2003). Vale ressaltar que tal política foi alterada a partir de 2016, sobretudo na composição dos dispositivos de saúde.

Porém, Centros de Atenção Psicossocial em Álcool e Outras Drogas (CAPSad) em cidades com mais de 100 mil habitantes, ainda é dentre as principais estratégias de acolhimento aos usuários de substâncias psicoativas, que tem a finalidade de desenvolver ações de caráter psicossocial voltadas ao tratamento dessa população na comunidade, por meio da construção de um projeto terapêutico que conta com uma equipe multiprofissional especializada e colaborativa dentro de uma perspectiva singularizada de evolução contínua, com a participação ativa do usuário do serviço e de acordo com as respectivas necessidades em saúde do indivíduo, tendo como uma de suas metas a inclusão social da pessoa com sofrimento psíquico (Brasil, 2003; Pinho et al., 2013. Carvalho et al, 2020).

Nesse caminho, a abordagem da Redução de Danos mostrou-se como uma alternativa de promoção da saúde, por olhar o usuário de SPA como um cidadão portador de direitos humanos. As estratégias não estão direcionadas para a abstinência, mas para a defesa da vida e o alcance da autonomia do usuário e de seus familiares, por meio de redes de suporte social, difusão da informação, educação e aconselhamento (Mangueira et al, 2015). 
A Organização Mundial da Saúde (OMS) (2019) destaca a importância da avaliação e do monitoramento da qualidade dos serviços de saúde mental, objetivando verificar a eficácia dos programas de prevenção e de tratamento de saúde mental. Nos CAPSad os usuários participam ativamente de seu tratamento, e suas avaliações subjetivas são consideradas como complemento dos resultados clínicos obtidos para melhorar a qualidade do cuidado, uma vez que eles estão diretamente envolvidos com o tratamento e são os principais interessados em melhorá-lo (Brasil, 2004).

Uma forma objetiva de mensurar se o usuário percebe mudanças em sua vida, após o início do tratamento em um CAPSad, é utilizando a Escala de Mudança Percebida (EMP) que permite avaliar resultados do tratamento, na perspectiva dos próprios usuários, demonstrando o impacto das intervenções em suas vidas (Bandeira et al, 2011).

A percepção de mudanças positivas pode ter um efeito potencializador, uma vez que proporciona ao usuário maior sentimento de controle sobre a própria vida (empoderamento), atestando a validade social da intervenção (Costa et al, 2008).

$\mathrm{Na}$ atualidade, as medidas de satisfação têm constituído o principal indicador de resultado da qualidade nos serviços comunitários de saúde mental (Mercier et al, 2004). Contudo, essas medidas são consideradas globais e abordadas em diferentes aspectos, sem focalizar os resultados e nem com a finalidade de gerar mudanças na vida dos usuários (Costa et al, 2008). A percepção dos usuários tem sido fundamental na avaliação dos resultados do tratamento na saúde mental (Brasil, 2003; Costa et al, 2008).

Nesse sentido, estudos internacionais indicam a importância dessa avaliação na perspectiva dos usuários nos serviços de saúde mental (Brasil, 2003; Costa et al, 2008). No entanto, no Brasil, os estudos que buscam avaliar a percepção de mudança decorrente do tratamento e da satisfação com os CAPSad dos usuários de substâncias psicoativas estão apenas no início.

No sentido de conhecer as repercussões da mudança percebida dos usuários em função do tratamento recebido em CAPSad, este estudo teve como objetivo avaliar a mudança percebida e identificar os fatores a ela associados em usuários dos Centros de Atenção Psicossocial em Álcool e Outras Drogas.

\section{Metodologia}

Trata-se de um estudo transversal destinado a avaliar dados referentes à estrutura, ao processo e aos resultados das práticas assistenciais desenvolvidas nos CAPSad, junto aos usuários. O referencial teórico-metodológico proposto foi o de avaliação tridimensional dos serviços de saúde mental (Donabedian, 1980), acreditando-se que, por abarcar as dimensões de estrutura, processo e resultado, possa propiciar a explicitação de questões referentes à satisfação e à mudança percebida no tratamento dos usuários. Este trabalho explora a dimensão resultado para analisar a percepção do usuário.

O estudo foi desenvolvido em 13 CAPSad sorteados, entre os 23 serviços existentes no Estado de Minas Gerais, no período de junho de 2014 a maio de 2015. A escolha dos CAPSad para a investigação foi feita de forma randomizada (aleatório simples) de um CAPSad por macrorregião e concentrações de serviços do Estado. O critério de inclusão dos CAPSad/macrorregião foi que estivessem credenciados pelo Ministério da Saúde e funcionando há pelo menos um ano.

A amostra dos participantes foi do tipo "aleatória simples", sem reposição, calculada com base em um estudo-piloto realizado em um CAPSad do município de São Paulo. O tamanho adequado da amostra foi estimado com base no valor do desvio padrão mais alto dos domínios do questionário da Escala de Satisfação dos Usuários com os Serviços de Saúde Mental (SATISBR) (Bandeira, Silva, 2012). Assim, para ter uma média da avaliação dos serviços estudados, calculou-se uma amostra de 360 usuários.

Os critérios de inclusão para os usuários dos CAPSad foram: pessoas com transtornos mentais graves e persistentes, decorrentes do uso e da dependência ao álcool e/ou outras drogas, com idade acima de 18 anos que estavam ativos nos serviços há mais de seis meses e que frequentam o serviço. Foram excluídos da amostra os usuários que tinham iniciado a participação nas atividades dos CAPSad há menos de seis meses. 
A amostra final foi composta por 330 usuários, com perda amostral de 60, visto que oito participantes recusaram, 14 estavam internados, quatro não apresentavam condições clínicas no período da coleta, 13 não compareceram à entrevista e 21 não puderam ser contatados mesmo após um mínimo de cinco tentativas (via profissionais do CAPSad, telefone e visita domiciliar), atitude caracterizada pelos serviços como abandono do tratamento.

O desfecho da mudança percebida em função do tratamento (variável dependente) foi definido a partir da aplicação da Escala de Mudança Percebida (EMP), entre os usuários por meio de entrevista estruturada. A escala EMP contém 19 itens quantitativos que avaliam como o paciente percebe, em geral, os efeitos surtidos desde o início do tratamento. As alternativas de resposta, para cada item, distribuem-se em uma escala tipo Likert de três pontos, sendo 1 para pior do que antes, 2 para sem mudança e 3 para melhor do que antes (Bandeira et al, 2011). A escala original foi recentemente renomeada como Perceived Improvement Interview por Perreault et al. (2010). No Brasil, essa escala foi validada e adaptada em 2011 por Bandeira et al. (2011) e apresenta elevada consistência interna $(\alpha=0,84)$.

As características da amostra do estudo foram analisadas resumindo-se as variáveis categóricas com números absolutos e porcentuais, e as variáveis quantitativas, utilizando-se médias e DP. Foi estimado o grau de mensuração do grau de satisfação global das escalas SATIS-BR e da EMP. Para verificar as associações entre as variáveis dependentes e independentes, foram elaborados modelos de regressão linear univariada e múltipla de mínimos quadrados ordinários (Ordinary Least Squares - OLS) (Baldi, Moore, 2013).

As variáveis independentes com $\mathrm{p} \leq 0,3$ na análise univariada foram incluídas no modelo multivariado, sendo mantidas no modelo final aquelas com $\mathrm{p} \leq 0,05$. As análises estatísticas foram realizadas utilizando-se o programa Statistical Package for the Social Sciences (SPSS), versão 20.

O estudo foi aprovado pelo Comitê de Ética da Escola de Enfermagem da Universidade de São Paulo (número 951.970/2015) e os princípios éticos assegurados de acordo com as Normas e Diretrizes Regulamentadoras da Pesquisa Envolvendo Seres Humanos - Resolução CNS 196/96, utilização de Termo de Consentimento Livre e Esclarecido (TCLE), garantia do direito de não participação em qualquer momento da pesquisa e anonimato do entrevistado.

\section{Resultados}

Participaram do estudo 330 usuários ( $85 \%$ do total de 390 usuários sorteados nos 13 CAPSad), sendo a maioria do sexo masculino (78,2\%); com faixa etária de 29 a 40 anos (33,9\%), resultando em uma média de idade de 44,8 anos, sem companheiro (70,6\%), em moradia regular $(89,1 \%)$, sem trabalho remunerado (16,4\%), sem escolaridade e com Ensino Fundamental incompleto $(54,5 \%)$, com renda familiar de até dois salários mínimos $(81,2 \%)$.

A prevalência de mudança percebida encontrada na amostra estudada foi de 86,1\% e a média global foi de 2,62 (para valores de 1 a 3). Mostraram-se estatisticamente associadas à mudança percebida, na análise univariada, as variáveis socioeconômicas sexo, escolaridade, renda familiar no último mês e bens duráveis ( $\mathrm{p} \leq 0,3)$ (Tabela 1). 
Tabela 1 - Análise univariada das variáveis socioeconômicas em relação à Escala de Mudança Percebida dos usuários da amostra dos Centros de Atenção Psicossocial de Álcool e Outras Drogas do Estado de Estado de Minas Gerais, Brasil, 2015 ( $\mathrm{n}=330$ ).

\begin{tabular}{|c|c|c|c|}
\hline Variáveis & $\bar{\beta}$ & $\begin{array}{l}\mathbf{r}^{2 *} \\
(\%)\end{array}$ & Valor de $p$ \\
\hline \multicolumn{4}{|l|}{ Idade (anos) } \\
\hline Total da amostra & 0,077 & 0,6 & $0,16 \dagger$ \\
\hline \multicolumn{4}{|l|}{ Sexo } \\
\hline Feminino & Referência & & \\
\hline Masculino & $-0,098$ & 1,0 & $0,07 \dagger$ \\
\hline \multicolumn{4}{|l|}{ Situação conjugal } \\
\hline Sem companheiro & Referência & & \\
\hline Com companheiro & 0,033 & 0,1 & 0,55 \\
\hline \multicolumn{4}{|l|}{ Escolaridade } \\
\hline Sem escolaridade ou com Ensino Fundamental incompleto & $-0,009$ & 0,8 & $0,00 \dagger$ \\
\hline $\begin{array}{l}\text { Ensino Fundamental completo ou ensino técnico/Médio } \\
\text { incompleto }\end{array}$ & $-0,002$ & & $0,00 \dagger$ \\
\hline Ensino Médio/curso técnico completo & 0,089 & & 0,97 \\
\hline \multicolumn{4}{|l|}{ Renda familiar no último mês, SM } \\
\hline$<2$ & $-0,003$ & 0,5 & $0,00 \dagger$ \\
\hline$\geq 2$ e $<4$ & 0,068 & & 0,95 \\
\hline \multicolumn{4}{|l|}{ Tipo de moradia atual } \\
\hline Moradia em situação vulnerável & Referência & & \\
\hline Moradia regular & 0,016 & 0,0 & 0,76 \\
\hline Bens duráveis & 0,072 & 0,5 & $0,19 \dagger$ \\
\hline
\end{tabular}

* R quadrado: coeficiente de determinação; † significância: $\mathrm{p} \leq 0,30$ - indica possibilidade de a variável ser incluída na regressão múltipla; $\$$ R\$ 788,00. SM: salários mínimos.

Fonte: Dados da pesquisa (2015).

A maior parte das variáveis clínicas apresentou correlação negativa com o item da escala EMP global. Segundo o modelo univariado, os usuários que consumiram droga ilegal no último mês, eram fumantes, usavam psicofármacos e passaram por internação e intercorrências durante o tratamento perceberam menor mudança decorrente do tratamento nos CAPSad. Por sua vez, os novos usuários, que se encontravam no início do tratamento, tinham ciência de seu diagnóstico e não tinham consumido bebida alcoólica no último mês, perceberam maior mudança decorrente do tratamento (Tabela 2). 
Tabela 2 - Análise univariada das variáveis clínicas em relação à Escala de Mudança Percebida dos usuários da amostra dos Centros de Atenção Psicossocial de Álcool e Outras Drogas do Estado de Minas Gerais, Brasil, 2015 (n= 330).

\begin{tabular}{|c|c|c|c|}
\hline Variáveis & $\boldsymbol{\beta}$ & $\begin{array}{l}\mathbf{r}^{2 *} \\
(\%)\end{array}$ & Valor de $p$ \\
\hline \multicolumn{4}{|c|}{ Internação psiquiátrica durante o tratamento no serviço } \\
\hline Não & Referência & & \\
\hline Sim & $-0,229$ & 5,2 & $0,00 \dagger$ \\
\hline \multicolumn{4}{|l|}{ Uso de tabaco } \\
\hline Não & Referência & & \\
\hline Sim & $-0,105$ & 1,1 & $0,05 \dagger$ \\
\hline \multicolumn{4}{|c|}{ Zonsumo de bebida alcoólica no último mês } \\
\hline Não & Referência & & \\
\hline Sim & 0,280 & 7,8 & $0,00 \dagger$ \\
\hline \multicolumn{4}{|l|}{ Consumo de droga ilegal no último mês } \\
\hline Não & Referência & & \\
\hline Sim & $-0,222$ & 4,9 & $0,00 \dagger$ \\
\hline \multicolumn{4}{|c|}{ Presença de outro problema de saúde (comorbidades) } \\
\hline Não & $-0,025$ & & 0,65 \\
\hline Sim & $-0,046$ & 0,2 & 0,41 \\
\hline \multicolumn{4}{|l|}{ Intercorrências durante o tratamento } \\
\hline Não & Referência & & \\
\hline Sim & $-0,200$ & 4,0 & $0,00 \dagger$ \\
\hline \multicolumn{4}{|l|}{ Uso de psicofármaco } \\
\hline Não & Referência & & \\
\hline Sim & $-0,088$ & 0,8 & $0,11 \dagger$ \\
\hline \multicolumn{4}{|l|}{ Primeira admissão no serviço } \\
\hline Não & Referência & & \\
\hline Sim & 0,145 & 2,1 & $0,00 \dagger$ \\
\hline \multicolumn{4}{|c|}{ Conhecimento sobre o diagnóstico médico } \\
\hline \multicolumn{4}{|l|}{ Não } \\
\hline Sim & $-0,082$ & 0,066 & $0,08 \dagger$ \\
\hline
\end{tabular}

* R quadrado: coeficiente de determinação; $†$ significância: $(\mathrm{p} \leq 0,30)$ : indica possibilidade de a variável ser incluída na regressão múltipla. Fonte: Dados da pesquisa (2015).

Todas as variáveis de processo e resultado foram incluídas no modelo de regressão múltipla e apresentaram correlação positiva com a EMP, exceto a variável "tem projeto terapêutico singular", a qual não se mostrou significativa, com $\mathrm{p} \geq 0,30$ (Tabela 3). 
Tabela 3 - Análises univariadas das variáveis de processo e resultado (escala Escala de Satisfação dos Pacientes com os Serviços de Saúde Mental - SATIS-BR) em relação à Escala de Mudança Percebida dos usuários da amostra dos Centros de Atenção Psicossocial de Álcool e Outras Drogas do Estado de Minas Gerais, Brasil, 2015 ( $\mathrm{n}=330$ ).

\begin{tabular}{|c|c|c|c|}
\hline Variáveis & $\boldsymbol{\beta}$ & $\begin{array}{l}\mathbf{r}^{2 *} \\
(\%)\end{array}$ & Valor de $p$ \\
\hline \multicolumn{4}{|c|}{ Considera a frequência no serviço suficiente } \\
\hline Não & Referência & & \\
\hline Sim & 0,30 & 9,0 & $0,00 \dagger$ \\
\hline \multicolumn{4}{|l|}{ Tem projeto terapêutico singular } \\
\hline \multicolumn{4}{|l|}{ Não } \\
\hline Sim & 0,050 & 0,3 & 0,36 \\
\hline \multicolumn{4}{|l|}{ Tem profissional técnico de referência } \\
\hline \multicolumn{4}{|l|}{ Não } \\
\hline Sim & 0,051 & 0,095 & $0,28 \dagger$ \\
\hline \multicolumn{4}{|c|}{ Participou de atividade em grupo no último mês } \\
\hline \multicolumn{4}{|c|}{ Não } \\
\hline Sim & 0,144 & 0,036 & $0,00 \dagger$ \\
\hline \multicolumn{4}{|c|}{ Participou de atividade na comunidade no último mês } \\
\hline \multicolumn{4}{|c|}{ Não } \\
\hline Sim & 0,090 & 0,040 & $0,06 \dagger$ \\
\hline Escore SATIS-BR Global & 0,366 & 13,4 & $0,00 \dagger$ \\
\hline
\end{tabular}

* R quadrado: coeficiente de determinação; $\uparrow$ significância: $(\mathrm{p} \leq 0,30)$ : indica possibilidade de a variável ser incluída na regressão múltipla. Fonte: Dados da pesquisa (2015).

A Tabela 4 apresenta os resultados da análise múltipla para a mudança percebida. Os resultados indicaram que o grau de percepção de melhora global foi maior entre as variáveis Ensino Médio ou curso técnico completo, frequentar o CAPSad, participar de atividade em grupo e satisfação global com o serviço $(\beta>0)$. Por outro lado, os entrevistados do sexo feminino, que consumiram bebida alcoólica no mês anterior à coleta de dados e que passaram por internação e intercorrências durante o tratamento demonstraram menor percepção de melhora decorrente da assistência $(\beta<0$; inversamente proporcional). O modelo final, o qual considerou o valor de $\mathrm{R}^{2}(0,341)$, atribuiu $34,1 \%$ da contribuição das variáveis investigadas ao grau de mudança percebida pelos usuários entrevistados (Tabela 4). 
Tabela 4 - Análise da regressão linear múltipla entre a mudança global percebida e as variáveis investigadas para o total da amostra de usuários dos Centros de Atenção Psicossocial de Álcool e Outras Drogas (CAPSad) do Estado de Minas Gerais, Brasil, $2015(\mathrm{n}=330)$.

\begin{tabular}{|c|c|c|c|}
\hline Variáveis & $\boldsymbol{\beta}$ & Erro padrão & Valor de $p$ \\
\hline \multicolumn{4}{|l|}{ Consumo de droga ilegal no último mês } \\
\hline Não & Referência & & \\
\hline Sim & $-0,068$ & 0,049 & 0,19 \\
\hline \multicolumn{4}{|l|}{ Considera a frequência no serviço suficiente } \\
\hline Não & Referência & & \\
\hline Sim & 0,152 & 0,046 & $0,00 *$ \\
\hline \multicolumn{4}{|l|}{ Sexo } \\
\hline Feminino & Referência & & \\
\hline Masculino & $-0,095$ & 0,041 & $0,04 \uparrow$ \\
\hline \multicolumn{4}{|l|}{ Escolaridade } \\
\hline Sem escolaridade ou com Ensino Fundamental incompleto & 0,020 & 0,041 & 0,69 \\
\hline $\begin{array}{l}\text { Ensino Fundamental completo ou ensino técnico/Médio } \\
\text { incompleto }\end{array}$ & 0,015 & 0,053 & 0,76 \\
\hline Ensino Médio/curso técnico completo & 0,123 & 0,083 & $0,01 *$ \\
\hline \multicolumn{4}{|l|}{ Renda familiar no último mês, SM } \\
\hline$<2$ & $-0,048$ & 0,050 & 0,35 \\
\hline$\geq 2$ e $<4$ & 0,057 & 0,122 & 0,25 \\
\hline Bens duráveis & 0,047 & 0,005 & 0,36 \\
\hline \multicolumn{4}{|l|}{ Primeira admissão no serviço } \\
\hline Não & Referência & & \\
\hline $\operatorname{Sim}$ & 0,049 & 0,035 & 0,32 \\
\hline \multicolumn{4}{|l|}{ Uso de psicofármaco } \\
\hline Não & Referência & & \\
\hline Sim & $-0,023$ & 0,045 & 0,64 \\
\hline \multicolumn{4}{|l|}{ Uso de tabaco } \\
\hline Não & Referência & & \\
\hline Sim & $-0,009$ & 0,038 & 0,85 \\
\hline \multicolumn{4}{|l|}{ Consumo de bebida alcoólica no último mês } \\
\hline Não & Referência & & \\
\hline Sim & $-0,186$ & 0.036 & $\leq 0,00^{*}$ \\
\hline \multicolumn{4}{|l|}{ Internação psiquiátrica durante o tratamento no CAPSad } \\
\hline Não & Referência & & \\
\hline Sim & $-0,137$ & 0,037 & $0,00 *$ \\
\hline \multicolumn{4}{|l|}{ Intercorrência (s) durante o tratamento } \\
\hline Não & Referência & & \\
\hline Sim & $-0,115$ & 0,039 & $0,02 \dagger$ \\
\hline Escore SATIS-BR Global & 0,272 & 0,042 & $\leq 0,00^{*}$ \\
\hline Idade & $-0,064$ & 0,002 & 0,20 \\
\hline \multicolumn{4}{|l|}{ Conhecimento sobre o diagnóstico médico } \\
\hline \multicolumn{4}{|l|}{ Não } \\
\hline $\operatorname{Sim}$ & $-0,082$ & 0,066 & 0,08 \\
\hline \multicolumn{4}{|l|}{ Tem profissional de referência dentro do CAPSad } \\
\hline Sim & 0,051 & 0,095 & 0,28 \\
\hline \multicolumn{4}{|l|}{ Participou de atividade em grupo no último mês } \\
\hline \multicolumn{4}{|l|}{ Não } \\
\hline Sim & 0,144 & 0,036 & $0,00 *$ \\
\hline \multicolumn{4}{|l|}{ Participou de atividade na comunidade no último mês } \\
\hline Não & & & \\
\hline Sim & 0,090 & 0,040 & 0,06 \\
\hline
\end{tabular}

*R Quadrado: 0,341. *Significante a $\mathrm{p} \leq 0,01 ; \dagger$ significante a $\mathrm{p} \leq 0,05$. SM: salário mínimo; SATIS-BR: Escala de Satisfação dos Pacientes com os Serviços de Saúde Mental.

Fonte: Dados da pesquisa (2015). 


\section{Discussão}

A ausência de estudos precedentes associando a percepção de mudanças ocorridas na vida de usuários em CAPSad serviços comunitários de álcool e outras drogas com as características do perfil sociodemográfico e clínico, além das dimensões do processo e da satisfação com o tratamento, limita o âmbito da discussão dos presentes resultados. Porém, torna-se uma oportunidade para discussão deste campo de investigação a respeito das percepções dos usuários em relação às mudanças percebidas com o tratamento recebido em CAPSad no estado de Minas Gerais, Brasil.

A média de mudança percebida identificada, de 2,62, aproxima-se da de outros estudos brasileiros. Neles, avaliou-se a mudança percebida em usuários inseridos em serviços de saúde mental, com amostras que caracterizaram um único serviço, e identificaram-se escores médios de percepção de melhora global de 2,46 e 2,94 com a escala EMP, o que indica que o tratamento oferecido pelos serviços de saúde mental tem dado resultados positivos (Cesari, Bandeira, 2010; Lima, 2013; Silva et al, 2012).

Esses resultados positivos podem ser explicados pelo fato de o instrumento enfocar principalmente fatos observáveis que ocorrem na vida dos usuários (Costa et al, 2011). Além disso, o quadro clínico moderado da amostra dos entrevistados pode ter favorecido uma maior adesão ao tratamento e, consequentemente, maior grau de percepção de melhora dos usuários.

Outro estudo que investigou os fatores associados à melhora percebida pelos usuários de CAPS no Sul do Brasil apontou resultado positivo quanto às mudanças percebidas pelos usuários desde sua inserção no serviço, relatando que $59 \%$ indicaram melhora (Franzmann, 2017).

Nas análises univariadas e múltipla da percepção de mudança dos usuários quanto ao perfil socioeconômico, destacaram-se as variáveis sexo e escolaridade. A variável sexo mostrou-se negativa, ao passo que a escolaridade foi positiva no modelo multivariado.

Os resultados dessas variáveis mostraram que as mulheres percebem menos mudança decorrente do tratamento nos CAPSad do que os homens, o que indica que a percepção pode divergir consideravelmente entre homens e mulheres em tratamento. Desse modo, sugere-se que a equipe multiprofissional responsável pelo cuidado dos usuários no território pela prestação de serviços promova tanto ações educativas e motivadoras, como um envolvimento maior das mulheres no tratamento, oferecendo grupos específicos para tratar questões relacionadas ao cotidiano das mulheres.

No que se refere à escolaridade, os usuários com maior nível de formação escolar (Ensino Médio ou curso técnico completo) apresentaram maior percepção de melhora decorrente do tratamento do que os entrevistados com escolaridade mais baixa ou considerados analfabetos.

Poucos estudos investigaram os fatores socioeconômicos e clínicos associados à maior percepção de mudanças pelos usuários em função do tratamento. No estudo realizado em Montreal, o qual se propôs a investigar a relação entre melhora percebida e satisfação do paciente em um programa de manutenção com metadona, não foi encontrada diferença significativa entre sexo e idade com pontuação global de mudança percebida em decorrência do tratamento (Perreault et al, 2010).

Em outro estudo realizado com usuários diagnosticados com transtornos não psicóticos, mostrou-se a influência do sexo na relação entre as mudanças percebidas e a satisfação com os serviços. No grupo de homens, foi observada uma correlação positiva entre a percepção de melhora, referente à capacidade de lidar com os problemas específicos e sintomas, e a satisfação com o serviço, enquanto para o sexo feminino obteve-se uma correlação positiva significativa das relações interpessoais e da satisfação com o serviço (Associação Brasileira de Psiquiatria, 2012).

O segundo modelo de análise de regressão univariada, o qual inclui as variáveis clínicas associadas à percepção de mudança global, apresentou relação significativa de menor percepção entre os usuários que consumiram drogas ilícitas no último mês por um mês, que eram fumantes, usavam psicofármacos, tiveram internação e intercorrências durante o tratamento, ao passo que os usuários que não consumiram bebida alcoólica no último mês perceberam maior mudança.

Não atingir a meta da abstinência pode influenciar negativamente na percepção de mudança do usuário. Estes resultados 
podem ser explicados pelo fato da recaída dos usuários de álcool e outras drogas ser devida às dificuldades enfrentadas desde o início do tratamento, visto que a maioria deles possui problemas sociais (como baixo nível de escolaridade, e dificuldade de resolver conflitos sociais e afetivos) e de saúde, tanto anteriores ao uso das substâncias como decorrentes dele (Associação Brasileira de Psiquiatria, 2012), além do objetivo do tratamento estar focado na abstinência e entender a recaída como mais um fracasso.

A menor percepção de mudanças percebidas pelos usuários dos CAPSad que tiveram maior número de internações psiquiátricas antes do tratamento no CAPSad, ou que foram internados ou apresentaram intercorrências durante o tratamento no CAPSad como no resultado desse estudo, pode demonstrar a dificuldade do indivíduo em perceber mudanças com o tratamento no território, tendo em vista que na atenção psicossocial o usuário é corresponsável pelo seu tratamento e o controle do uso de drogas não é respondido com o afastamento do mesmo do seu contexto de vida.

Nesse sentido, torna-se fundamental trabalhar com os usuários dos CAPSad na perspectiva da Redução de Danos que entende a recaída como parte do processo e respeita o direito que o sujeito tem de não conseguir ou não querer parar de usar substâncias.

Não foram encontrados, na literatura, estudos que relacionassem o processo de internação e intercorrências durante o tratamento com a mudança percebida na área da dependência química, o que sugere a originalidade deste estudo.

Investigações sobre a relação entre complicações e intercorrências em usuários de substâncias psicoativas e percepção de mudança ainda são escassas. Conhecer essas relações é importante para a elaboração de políticas públicas voltadas para o consumo de substâncias psicoativas, no sentido de melhorar as intervenções quanto aos níveis de gravidade da dependência química e, com isso, gerar mudanças no processo terapêutico.

Em relação ao processo e ao resultado do tratamento, as variáveis incluídas no modelo multivariado que mostraram nível de significância de 30\% foram primeira admissão, considerar a frequência suficiente no CAPSad, ter profissional de referência, participação de atendimento em grupos no CAPSad, participação em atividades na comunidade e satisfação global, associadas a uma percepção positiva de mudança.

Estudos internacionais e nacionais similares a este também apresentaram as mudanças percebidas pelos usuários em função do tratamento em serviços de saúde mental como resultado, tendo a variável mais preditiva da satisfação global com os serviços, confirmando a correlação positiva entre essas duas variáveis (Hasler et al, 2004; Perreault et al, 2010; Bandeira et al, 2011; Andrade et al, 2012; Costa et al, 2011; Cesari, Bandeira, 2012; Silva et al, 2012; Franzmann et al, 2017).

Esses resultados mostram a relevância do uso do escore de mudança percebida, sob o ponto de vista da satisfação dos usuários com a equipe, as condições gerais do serviço e a qualidade do atendimento. Estudos também têm mostrado a importância de se explorarem as relações dessas variáveis no tratamento do abuso de substâncias (Perreault et al, 2010; Lima, 2013).

A satisfação costuma ser investigada enquanto variável dependente da mudança percebida. No presente estudo, optouse por analisar a correlação contrária, no intuito de verificar a influência de aspectos subjetivos, como a satisfação, na percepção de mudança. A satisfação com os serviços tem sido utilizada há mais de 20 anos como desfecho primário na perspectiva dos usuários, ao passo que a percepção de melhora é recente e oferece uma nova visão da avaliação de programa do ponto de vista dos pacientes. Estudos futuros, que envolvam o consumo de substâncias psicoativas e as condições gerais de vida (saúde física, mental e qualidade de vida) devem ser considerados na avaliação global dos resultados quanto ao tratamento. O mesmo se aplica às características do perfil dos indivíduos, para que se obtenha uma melhor compreensão dos fatores envolvidos na melhoria percebida (Perreault et al, 2010).

É possível que tais associações se devam à frequência de participação e ao contato dos usuários com as atividades oferecidas pela equipe nos CAPSad, demonstrando que tais variáveis podem contribuir para um melhor estado de saúde geral, refletindo na percepção das mudanças percebidas pelos usuários do serviço. Tanto esses dados como a satisfação global dos 
usuários podem sugerir bons indicadores de avaliação dos resultados do tratamento, bem como confiança por parte do usuário nos serviços de saúde comunitários especializados no tratamento de substâncias psicoativas, como o CAPSad no contexto brasileiro (Perreault et al, 2010, Carvalho et al, 2020).

No estudo realizado em Montreal, por sua vez, não foram apontadas associações entre a percepção de mudança e a frequência do tratamento por meio de um programa de manutenção com metadona, sugerindo que seria pertinente realizar investigações longitudinais que mensuraram a melhora percebida em diferentes fases do tratamento, uma vez que, com suas atividades diárias avaliadas no serviço em diferentes momentos, os usuários não estariam mais centrados apenas no uso de drogas, mas em outras atividades de vida diária. Portanto, altos escores na EMP poderiam ser explicados por mudanças na situação global (Perreault et al, 2010).

Desse modo, os dados escassos da literatura não permitiram maior aprofundamento das discussões a seguir, as quais avaliam a percepção de mudança pelos próprios usuários submetidos a tratamento para substâncias psicoativas, com relação às variáveis de processo e de satisfação com os serviços.

Este estudo possui limitações no que se refere à possibilidade de generalizações dos seus resultados, tendo em vista os possíveis casos de abandono do serviço e de perdas de participantes. Muitos dos sorteados não puderam ser contatados, e eles podem ter limitado a amostra aos casos com resultados mais positivos.

Destaca-se, ainda, a limitação dos estudos transversais, que se vale de entrevistas como principal fonte de dados, uma vez que estão sob a ação de fatores como memória e influência de acontecimentos recentes na vida dos indivíduos. Além disso, por se tratar de um estudo correlacional, é necessário ter cautela ao interpretar os resultados, uma vez que o estudo transversal não permite o estabelecimento de relações causais entre as variáveis estudadas. Pesquisas futuras, do tipo longitudinal, poderiam acompanhar a evolução da percepção de mudança de pacientes nas diferentes fases do tratamento, identificando a sequência das percepções de mudanças em diferentes aspectos da vida diária, em função do tratamento recebido (Perreault et al, 2010; Costa et al, 2011; Silva et al, 2012).

\section{Conclusão}

A análise dos fatores associados aos construtos avaliados indicou forte associação de variáveis socioeconômicas, clínicas, do processo e de satisfação global na determinação da mudança percebida pelos usuários, a partir dos quais deve ser possível refletir e propor ações de caráter coletivo para auxiliar os serviços no provimento de tratamentos mais adequados às necessidades dos usuários. Além disso, os resultados sugerem possibilidades de melhoria no cuidado, visando a modificações que podem influenciar no envolvimento do tratamento aos usuários de substâncias psicoativas.

Pode-se, portanto, afirmar que a hipótese apresentada no presente estudo foi confirmada, tendo sido encontrada uma relação significativa entre as mudanças percebidas em função do tratamento e as variáveis sexo, escolaridade, consumo de bebida alcoólica no último mês, internação psiquiátrica, intercorrência, frequência ao CAPSad, participação em grupo terapêutico e satisfação global com o CAPSad medida pelos usuários.

O presente estudo somou esforços para a consolidação das políticas de enfrentamento das drogas lícitas e ilícitas, dentre as quais a redução de danos e o desenvolvimento das relações de vínculo e de responsabilização entre as equipes e a população adstrita no território, garantindo a melhoria da qualidade do serviço e a longitudinalidade do cuidado. Os fatores aqui descritos, relacionados ao processo e ao resultado, podem servir como indicadores para investigações futuras que atendam às especificidades de uma área ainda recente. 


\section{Referências}

Andrade, M. C., Bandeira, M. Perreault, M., Angelico, A. P., Oliveira, M. S. (2012). The relationship between symptomatic changes and perceived improvement among patients treated in Brazilian community mental health services. Psychiatr. Q., 83 (3), 361-70. doi: 10.1007/s11126-012-9206-6

Associação Brasileira de Psiquiatria (APA). (2012). Abuso e dependência: crack. Rev. Assoc. Med. Bras., $58 \quad$ (2), 138-40. https://www.scielo.br/pdf/ramb/v58n2/v58n2a08.pdf

Baldi, B., Moore, D. S. (2013). The practice of statistics in the life sciences. 3rd ed. New York: WH Freeman.

Bandeira, M. Andrade, M. C., Costa, C. S., Silva, M. A. (2011). Patient's perception on the treatment in mental Health services: validating the Perception of Change Scale - patient version. Psicol. Reflex. Crit., 24 (2), 236-44. doi: https://doi.org/10.1590/S0102-79722011000200004

Bandeira, M., Silva, M. A. (2012). Patients' Satisfaction with Mental Health Services Scale (SATIS-BR): validation study. J Bras Psiquiatr., 61 (3), 124-32. doi: https://doi.org/10.1590/S0047-20852012000300002

Brasil. Ministério da Saúde. (2003). A Política do Ministério da Saúde para atenção integral a usuários de álcool e outras drogas. Retrieved from: http://bvsms.saude.gov.br/bvs/publicacoes/politica_atencao_alcool_drogas.pdf

Carvalho, M. F. A. A., Coelho, E. A. C., Oliveira, J. F., Medeiros, A. T., Barros, A. R. \& Freire, A. K. S. (2020). Care in psychosocial care enhancing subjectivities of people who use drugs. Research, Society and Development, 9(7): 1-22, e113974002. doi: http://dx.doi.org/10.33448/rsd-v9i7.4002

Cesari, L., Bandeira, M. (2010). Assessment of quality of life and perception of change in patients with schizophrenia. J. Bras Psiquiatr. 59 (4), 293-301. doi: https://doi.org/10.1590/S0047-20852010000400005

Costa, C. S., Bandeira, M., Cavalcanti, R. L., Scalon, J. D. (2011). Perceptions by patients and families towards treatment outcomes in mental health services. Cad. Saúde Pública, 27 (5), 995-1007. doi: https://doi.org/10.1590/S0102-311X2011000500017

Donabedian, A. (1980). Explorations in Quality Assessment and Monitoring. The Definition of quality and approaches to its assessment. Ann Arbor, MI: Health Administration Press.

Franzmann, U. T., Kantorski, L. P., Jardim, V. M. da R., Treichel, C. A. dos S., Oliveira, M. M. de P., Fabiane, M. (2017). Fatores associados à percepção de melhora por usuários de Centros de Atenção Psicossocial do Sul do Brasil. Cad. Saúde Pública, 33 (7), e00085216. doi: https://doi.org/10.1590/0102$311 \mathrm{X} 00085216$

Hasler, G., Moergeli, H., Bachmann, R., Lambreva, E., Buddeberg, C., Schnyder, U. (2004) Patient satisfactions with outpatient psychiatric treatment: The role of diagnosis, pharmacotherapy, and perceived therapeutic change. Can. J. Psychiatry, 49 (5), 315-21. doi: 10.1177/070674370404900507.

Lapischies, S. R., Jardim, V. M., Kantorski, L. P. (2014). Factors associated with satisfaction at work in Psychosocial Care Centers. Rev Latino-Am Enfermagem, 22 (6), 950-58. doi: https://doi.org/10.1590/0104-1169.3474.2500

Lima, F. C. (2013). Avaliação da mudança percebida e da satisfação de usuários de CAPS-AD. (Dissertação de Mestrado). Centro de Filosofia e Ciências Humanas, Universidade Federal de Santa Catarina, Florianópolis, Brasil.

Mangueira, S. O., Guimarães, F. J., Mangueira, J. O., Fernandes, A. F., Lopes, M. V. (2015). Health promotion and public policies of alcohol in Brazil: integrative literature review. Psicol Soc., 27 (1), 157-68. doi: https://doi.org/10.1590/1807-03102015v27n1p157

Mercier, L., Landry, M., Corbiere, M., Perreault, M. (2004) Measuring client's perception as outcome measurement. In: Roberts A. R., Yeager, K. R., editors. Evidence-based practice manual: research and outcome measures in health and human services. Oxford: Oxford University Press, p. $904-9$

Miranda, P. O., Souza, O. F., Ferreira, T. F. (2014). Evaluation of satisfaction of patients and relatives in a mental health service in the city of Rio Branco Acre. J. Bras. Psiquiatr., 63 (4), 332-40. doi: https://doi.org/10.1590/0047-2085000000042

Perreault, M., White, N. D., Fabres, E., Landry, M., Anestin, A., Rabouin, D. (2010). Relationship between perceived improvement and treatment satisfaction among clients of a methadone maintenance program. Eval Program Plann. (33), 410-7. doi: 10.1016/j.evalprogplan.2009.12.003

Pinho, P. H., Oliveira, M. F., Claro, H. G. Pereira, M. O., Almeida., M. M. (2013). A Concepção dos profissionais de saúde acerca da reabilitação psicossocial nos eixos: morar, rede social e trabalho dos usuários de substâncias psicoativas. Revista Portuguesa de Enfermagem de Saúde Mental, (9), 29-35. http://www.scielo.mec.pt/scielo.php?script=sci_arttext\&pid=S1647-21602013000100005\&lng=pt\&nrm=iso\&tlng=pt

Silva, M. A., Bandeira, M., Scalon., J. D., Quaglia., M. A. (2012). Patients' satisfaction with mental health services: the perception of changes as predictor. J. Bras. Psiquiatr., 61(2):64-71. Doi: https://doi.org/10.1590/S0047-20852012000200002

World Health Organization (WHO). (2019). Global Status Report on Alcohol and Health 2018. https://www.who.int/publications/i/item/9789241565639 